\title{
¿Rendimiento en exámenes o promedio general? Algunas cuestiones sobre la medición del rendimiento académico en investigación
}

Sergio Domínguez-Lara*

Magíster en Psicología Clínica y de la Salud. Docente investigador. Universidad de San Martín de Porres, Lima, Perú. Correo electrónico: sdminguezl@usmp.pe

Recibido: 21 de julio del 2016

Aprobado: 16 de diciembre del 2016

Cómo citar este artículo: Domínguez-Lara, S. (2017). ¿Rendimiento en exámenes 0 promedio general? Algunas cuestiones sobre la medición del rendimiento académico en investigación. Pensando Psicología, 13(21), 33-39. doi: http://dx.doi.org/10.16925/ pe.v13i21.1712

\section{Resumen}

Introducción: el rendimiento académico (RA) es un tema relevante en la investigación educativa, pero su operacionalización aún está sujeta a controversia, ya que no hay un criterio unificado sobre considerar el promedio ponderado (PP), o el rendimiento en los exámenes (RE), como medida de RA en investigación, sobre todo con constructos dependientes de la situación, como, por ejemplo, la ansiedad ante exámenes (AE). Objetivo: el objetivo fue analizar la equivalencia empírica entre el PP y el RE. Método: participaron 115 estudiantes de psicología de una universidad privada con edades entre los 18 y 31 años $(M=22,704 ; D E=3,126)$, pertenecientes a tres ciclos académicos distintos. Fueron evaluados con el Inventario de Ansiedad ante Exámenes-Estado, considerando además las calificaciones de exámenes y promedios ponderados de cada estudiante. En cuanto al análisis de datos, fueron implementadas correlaciones bivariadas entre RE y PP $\left(r_{\mathrm{RE}-\mathrm{PP}}\right)$. Posteriormente, las $r_{\mathrm{RE}-\mathrm{PP}}$ observadas en diferentes ciclos fueron comparadas. Por último, se analizaron las diferencias entre las correlaciones de la AE con el RE $\left(r_{\mathrm{AE}-\mathrm{RE}}\right)$ y el PP $\left(r_{\mathrm{AE}-\mathrm{PP}}\right)$, observadas en cada ciclo. Todos los análisis fueron complementados con una medida de magnitud del efecto. Resultados: la correlación entre RE y PP es de magnitud baja, y las diferencias halladas entre correlaciones no fueron estadísticamente significativas. Conclusiones: no es conveniente considerar como equivalentes el RE y el PP en el marco de estudios con variables dependientes de la situación. Se sugirió la importancia de superar las limitaciones observadas en el presente estudio.

Palabras clave: ansiedad ante exámenes, estudiantes universitarios, promedio académico, rendimiento académico. 


\title{
Test performance or general average? Some questions about measuring academic performance in research
}

\begin{abstract}
Introduction: Academic performance (AP) is a relevant subject in educational research, but its operationalization is still subject to controversy since there is no unified opinion on considering weighted average (WA) or test performance (TP) as a measure of AP in research, especially with situation-dependent constructs such as test anxiety (TA). Objective: The objective was to analyze empirical equivalence between WA and TP. Method: 115 psychology students from a private university with ages between 18 and $31(M=22,704 ; S D=3,126)$ belonging to three different academic cycles participated. They were evaluated with the State-Test Anxiety Inventory, also considering test scores and weighted averages of each student. Regarding data analysis, bivariate correlations between TP and WA $\left(r_{\text {TP-WA }}\right)$ were implemented. Subsequently, the $r_{\text {TP-WA }}$ observed in different cycles were compared. Finally, we analyzed differences between TA-TP $\left(r_{\text {TA-TP }}\right)$ and TA-WA $\left(r_{\text {TA-WA }}\right)$ correlations observed in each cycle. All analyses were complemented with a magnitude measure of the effect. Results: The correlation between TP and WA is of low magnitude, and the differences found between correlations were not statistically significant. Conclusions: It is not convenient to consider TP and wA as equivalent in the context of studies with situation-dependent variables. The importance of overcoming the limitations observed in this study was suggested.
\end{abstract}

Keywords: test anxiety, university students, academic average, academic performance.

\section{Rendimento em provas ou média geral? Algumas questões sobre a medição do rendimento acadêmico em pesquisa}

\section{Resumo}

Introdução: o rendimento acadêmico (RA) é um tema relevante na pesquisa em educação, mas sua operacionalização ainda está sujeita à controvérsia, já que não há um critério unificado sobre considerar a média ponderada (MP), ou o rendimento nas provas (RP), como medida de RA em pesquisa, principalmente com construtos dependentes da situação, como, por exemplo, a ansiedade diante de provas (AP). Objetivo: o objetivo foi analisar a equivalência empírica entre o MP e o RP. Método: participaram 115 estudantes de psicologia de uma universidade particular entre 18 e 31 anos $(M=22,704 ; D E=$ $3,126)$, pertencentes a três ciclos acadêmicos diferentes. Foram avaliados com a Escala de Ansiedade diante de Provas-Estado, considerando ainda as qualificações de provas e MP de cada estudante. Quanto à análise de dados, foram implantadas correlações bivariadas entre RP e MP $\left(r_{\text {RP-MP }}\right)$. Em seguida, as $r_{\text {RP-MP }}$ observadas em diferentes ciclos foram comparadas. Por último, analisaram-se as diferenças entre as correlações da AP com o RP $\left(r_{\text {AP-RP }}\right)$ e o MP $\left(r_{\text {AP-MP }}\right)$, observadas em cada ciclo. Todas as análises foram complementadas com uma medida de magnitude de efeito. Resultados: a correlação entre RP e MP é de magnitude baixa, e as diferenças encontradas entre correlações não foram estatisticamente significativas. Conclusões: não é conveniente considerar como equivalentes o RP e o MP no âmbito dos estudos com variáveis dependentes da situação. Sugeriu-se a importância de superar as limitações observadas no presente estudo.

Palavras-chave: ansiedade diante de provas, estudantes universitários, média acadêmica, rendimento acadêmico. 


\section{Introducción}

El rendimiento académico (RA) es un constructo complejo (Edel, 2003), pues no existe un acuerdo unánime respecto a su definición conceptual. Frecuentemente, se le considera como el logro alcanzado por el estudiante luego de un proceso de instrucción (Aiken, 1996), o como la cantidad de aprendizajes obtenidos (Pizarro, Clark y Allen, 1987), usualmente a partir de diferentes tipos de evaluación (Aiken, 1996, Cortada de Kohan, 1999), los cuales se reflejan en indicadores cuantitativos (e. g. calificaciones). Esto incluye, además, el proceso comparativo con personas de la misma edad o nivel educativo (Jiménez, 1994).

En este sentido, el RA puede ser operacionalizado, tanto con el promedio general de calificaciones (promedio ponderado, PP), como con el rendimiento en exámenes (RE). Por un lado, el PP es la síntesis del rendimiento del estudiante a lo largo de su vida universitaria, e incluye evaluaciones periódicas, intermedias y de final de ciclo, trabajos grupales, asistencias o exposiciones; es decir, un reflejo de su conducta académica que podría, en última instancia, predecir la deserción estudiantil (Barrero, Garzón y Gómez, 2013). Por su parte, el RE obedece a un rendimiento circunscrito solo a un evento: el examen. De este modo, ambas formas de evaluar el RA pueden ser observadas en las investigaciones que analizan su relación con otros constructos de interés, y las consideran como medidas de RA de forma intercambiable.

Esto no supondría dificultad si el PP, en representación del RA, se relacionara con constructos académicos de amplio espectro como los estilos de aprendizaje (e. g. Vázquez, Noriega y García, 2013), o el aprendizaje auto-regulado (e. g. Vázquez y Daura, 2013) que, por definición, abarcan una amplia gama de contextos de aprendizaje y desempeño compatibles con la gama de tareas y situaciones mencionados en el párrafo anterior. No obstante, cuando el PP es asociado a constructos vinculados a una situación particular (e. g. los exámenes), no se tiene en cuenta que el pP se basa en una serie de aspectos que superan la situación específica y sería, por lo menos, inaplicable a dicha circunstancia.

Un caso particular es el de los estudios que relacionan el RA con la ansiedad ante exámenes (AE). La AE es un rasgo de personalidad específico para exhibir ansiedad de forma más intensa y frecuente de lo habitual, con preocupaciones que interfieren con la atención, la concentración y la realización de exámenes (Araki, Iwawaki y Spielberger, 1992; Spielberger 1980; Spielberger y Vagg, 1995). Así, entonces, la AE es un constructo altamente dependiente de la situación evaluativa, por lo cual sería coherente en estos estudios que impliquen situaciones vinculadas directamente al momento de la evaluación, de modo que el RE sea la medida idónea de RA. Sin embargo, algunas investigaciones que asocian la AE con el RA utilizan el PP (o sus equivalentes en distintos sistemas educativos, tales como el Grade Point Average [GPA]) como medida de RA, sin demostrar la equivalencia empírica entre el PP y el RE (Chapell et al., 2005; DordiNejad et al., 2011; Khalaila, 2015; Akram y Mahmood, 2010; Shakir, 2014); mientras que otros sí consideran el RE (Cassady y Johnson, 2001; Tooranposhti, 2011), o incluso no establecen distinción alguna al momento de reportar los resultados (Seipp, 1991).

Esta situación es crítica, ya que en ocasiones se reportan resultados contradictorios: en algunos casos se relacionan AE y RA, y en otros no; pero esas correlaciones podrían estar influidas por el tipo de indicador de RA utilizado (PP O RE). En síntesis, los autores de los estudios citados anteriormente parecen no diferenciar que ambos aspectos (PP o RE) no podrían ser intercambiables en la evaluación de un constructo como la AE.

De este modo, el objetivo del presente estudio fue evaluar empíricamente la relación entre la RE y el PP mediante diversos métodos. El primero, con base en correlaciones bivariadas entre RE y PP $\left(r_{\mathrm{RE}-\mathrm{PP}}\right)$; el segundo, comparando las correlaciones $\left(r_{\text {RE-PP }}\right)$ observadas en diferentes grupos; y el último, analizando las diferencias entre las correlaciones de la $\mathrm{AE}$ con la $\mathrm{RE}\left(r_{\mathrm{AE}}\right.$ $\left.{ }_{\mathrm{RE}}\right)$ y el PP $\left(r_{\mathrm{AE}-\mathrm{PP}}\right)$ en estudiantes universitarios limeños (Perú).

Conociendo que la mayoría de estudios asumen con fines de investigación la equivalencia conceptual y empírica entre la RE y el PP como medida de RA, la hipótesis principal apunta a que $r_{\mathrm{RE}-\mathrm{PP}}$ es de magnitud elevada y no difiere a través de los grupos, y además que $r_{\mathrm{AE}-\mathrm{RE}} \mathrm{y} r_{\mathrm{AE}-\mathrm{PP}}$ son estadísticamente similares.

Este estudio es relevante en tanto proveerá de evidencia que facilitará las decisiones de los investigadores sobre la pertinencia del uso de uno u otro recurso (PP O RE), como medida de RA en estudios con constructos de orden situacional.

\section{Método}

Este estudio metodológico (Ato, López y Benavente, 2013) contó con la participación de una muestra de 115 estudiantes universitarios $(86,1 \%$ mujeres $)$, con edades comprendidas entre los 18 y 31 años $(M=$ $22,704 ; D E=3,126)$, pertenecientes al cuarto $(\mathrm{n}=37)$, séptimo $(n=32)$ y octavo ciclo $(n=46)$ de la carrera 
profesional de psicología de una universidad privada ubicada en Lima Metropolitana (Perú).

\section{Instrumento}

Inventario de Ansiedad ante Exámenes-Estado o TAI-Estado (Domínguez-Lara, 2016a). Está compuesto por 15 ítems que evalúan de forma unidimensional la AE con cuatro alternativas de respuesta que reflejan intensidad ("Nada", "Algo", "Bastante" y "Mucho"). Los indicadores de confiabilidad fueron adecuados $(\alpha=0,932$; IC95\% 0,903-0,943).

\section{Procedimiento}

Los estudiantes universitarios participantes fueron evaluados con el TAI-Estado después de rendir el examen final, indicándoles que respondan con base en cómo se sintieron durante el mismo. Se les explicó que la evaluación formaba parte de una investigación llevada a cabo en la facultad, que su participación era voluntaria y sus respuestas serían tratadas de forma confidencial. Al término, se les agradeció por su colaboración. Las calificaciones de los exámenes fueron obtenidas directamente de los docentes que evaluaron, y los promedios ponderados de cada estudiante fueron obtenidos mediante una solicitud a la oficina responsable.

\section{Análisis de datos}

Inicialmente, se implementaron correlaciones bivariadas entre RE y PP $\left(r_{\text {RE-PP }}\right)$ mediante el coeficiente de Pearson, tanto de forma general (la muestra completa), como al interior de cada grupo (ciclo de estudios). Posteriormente, las correlaciones $\left(r_{\mathrm{RE}-\mathrm{PP}}\right)$ observadas en diferentes grupos fueron comparadas utilizando una prueba ómnibus de comparación múltiple (Paul, 1989), haciendo uso del programa INCOR (Silver, Zaikina, Hittner y May, 2008) que se basa en la prueba $\chi_{c(\mathrm{~F})}^{2}$, de forma conjunta con una prueba de rangos a posteriori, usando los grados de libertad del enfoque Fisher-Hayter. Esta es la principal ventaja de este procedimiento que controla eficientemente el error tipo I cuando se comparan más de dos correlaciones (Silver y Merino-Soto, 2016).

Finalmente, fueron analizadas las diferencias entre las correlaciones de la AE con el RE $\left(r_{\mathrm{AE}-\mathrm{RE}}\right)$ y el PP $\left(r_{\mathrm{AE}-\mathrm{PP}}\right)$, de forma general y en cada ciclo, usando el programa COMPCOR (Silver, Ullman y Picker, 2015). El análisis estuvo basado en un método que permite comparar dos correlaciones independientes con un elemento en común (en este caso, la $\mathrm{AE}$ ), obteniendo un intervalo de confianza para las diferencias $\left({ } C_{d i f}\right)$, concluyendo que estas son significativas si no incluye al cero (Silver y Merino, 2016).

Debido a que todos los análisis mencionados anteriormente están influidos por el tamaño muestral, se complementaron con una medida de magnitud del efecto, a fin de valorar la fuerza de la correlación entre las variables: 0,20 (mínimo aceptable), 0,50 (moderado) y 0,80 (fuerte) (Ferguson, 2009); así como para la diferencia de correlaciones mediante el estadístico $q$ (Cohen, 1992): 0,10 (diferencia pequeña), 0,30 (diferencia moderada), y 0,50 (diferencia grande).

\section{Resultados}

Las correlaciones entre el RE y el PP en los diferentes grupos se observan en la tabla 1, así como la diferencia entre las mismas. De forma sintética, la relación entre RE y PP es relativamente baja en el grupo completo, y de carácter diferencial entre los diferentes grupos. Asimismo, no existen diferencias estadísticamente significativas entre $r_{\mathrm{AE}-\mathrm{RE}}$ y $r_{\mathrm{AE}-\mathrm{PP}}$, así como tampoco diferencias con significancia práctica (q alrededor de 0,10; Cohen, 1992).

\section{Discusión}

La hipótesis principal defendía la equivalencia entre el RE y el PP, es decir, que podrían utilizarse de forma intercambiable como medidas de RA con base en la literatura previa, la cual parece no hacer distinción entre ambas. De acuerdo con Ferguson (2009), esto suponía que las correlaciones observadas fueran elevadas $(r>$ $0,80)$, y si bien alcanzan significancia práctica $(r>0,20)$, las magnitudes no son lo suficientemente grandes. Por tal motivo, RE y PP no podrían ser consideradas como medidas equivalentes del RA, ya que, tal como se indicaba al principio, están enfocados en aspectos muy distintos, y eso se refleja en sus coeficientes de correlación.

La significancia práctica en correlación hace referencia al porcentaje de varianza compartida entre las medidas. Dicho de otro modo, al grado en que dos mediciones son similares. En este caso, se esperaría un solapamiento $(r>0,80)$ que no se dio, por lo que es difícil sostener que son medidas intercambiables. Por ello, es necesario tener esto en cuenta cuando sean estudiados constructos dependientes de la situación.

Otro aspecto a resaltar es que dicha relación $\left(r_{\text {RE-PP }}\right)$ es distinta en los diferentes grupos de estudiantes, quienes pertenecen a diferentes ciclos y, por ende, fueron 
Tabla 1

Análisis de las correlaciones entre calificación en el examen, promedio ponderado y ansiedad ante exámenes

\begin{tabular}{|c|c|c|c|c|c|c|}
\hline & $r_{\text {RE-PP }}$ & $\chi_{c(\mathrm{~F})}^{2}$ & $r_{\mathrm{AE}-\mathrm{RE}}$ & $r_{\mathrm{AE}-\mathrm{PP}}$ & $I C_{d i f}$ & $q$ \\
\hline General & $0,252^{\star \star}$ & - & $-0,167$ & $-0,359$ & $\begin{array}{c}-0,022 \\
0,401\end{array}$ & 0,179 \\
\hline Cuarto ciclo & $0,469^{* *}$ & & $\overline{-}^{-}$ & $-0,322$ & $\begin{array}{c}-0,200 \\
0,423\end{array}$ & 0,099 \\
\hline Séptimo ciclo & $-0,029$ & $17,594^{\star *}$ & $-0,200$ & $-0,314$ & $\begin{array}{c}-0,585 \\
0,372\end{array}$ & 0,107 \\
\hline Octavo ciclo & $0,378^{\star *}$ & & $-0,324^{*}$ & $-0,443^{* *}$ & $\begin{array}{c}-0,416 \\
0,178\end{array}$ & 0,103 \\
\hline
\end{tabular}

Nota. ${ }^{*}: p<0,05 ;{ }^{* *}: p<0,01 ; r_{\mathrm{RE}-\mathrm{PP}}$ : correlación entre el rendimiento en el examen y el promedio ponderado; $r_{\mathrm{AE}-\mathrm{RE}}$ : correlación entre la ansiedad ante exámenes y el rendimiento en el examen; $r_{\mathrm{AE}-\mathrm{pP}}$ : correlación entre la ansiedad ante exámenes y el promedio ponderado; $I C_{d i f}$ intervalo de confianza para las diferencias; $q: q$ de Cohen. Elaboración propia.

evaluados en exámenes cuyo contenido fue distinto. Esto agrega evidencia adicional a investigaciones que abogan sobre la necesidad de contextualizar en asignaturas determinadas la relación entre medidas, dado que esta tiende a ser diferente entre ellas (Domínguez-Lara, Calderón-de la Cruz, Alarcón-Parco y Navarro-Loli, en prensa). Es decir, no solo basta con saber qué medida de RA usar, sino en qué contexto evaluativo (e. g. asignatura) se emplea, dado que múltiples factores pueden afectar la dinámica de clase (Sánchez-Rosas, Takaya y Molinari, 2016).

De este modo, cada contexto evaluativo incluiría aspectos propios como la dificultad percibida (e. g., los cursos de estadística o metodología de la investigación se perciben habitualmente como de mayor dificultad), interacción con los pares (es más probable compartir experiencias de aprendizaje en algunas asignaturas en particular), e incluso el estilo de enseñanza del docente, quien podría llevar a interpretar la situación evaluativa como una situación desafiante o amenazante. Esos elementos hacen que la dinámica de clase en cada asignatura sea única, incluso en el mismo ciclo académico, posiblemente influyendo en la forma de manifestarse de algunos constructos (e. g. AE). Por ello, es necesario conocer cuál es el tipo de interacción predominante en cada asignatura, así como evaluar y analizar los constructos de interés al interior de cada una.

Esos resultados podrían entrar en contradicción con el hallazgo que indica que las correlaciones entre la AE, la RE $\left(r_{\mathrm{AE}-\mathrm{RE}}\right)$ y el PP $\left(r_{\mathrm{AE}-\mathrm{PP}}\right)$ son estadísticamente similares. Sin embargo, al tener en cuenta que la varianza compartida entre el RE y el PP es baja, dicha similitud pudo estar influida por factores ajenos a dichas variables per se como, por ejemplo, el tamaño muestral que hace más probable la aceptación de la hipótesis nula, referida a la igualdad estadística de correlaciones (Domínguez-Lara, 2016b).

A pesar de que a la fecha no existen investigaciones previas que aborden este tema, los resultados se constituyen como una alerta a los investigadores, porque si no se tienen en cuenta las diferencias intrínsecas entre el RE y el PP se puede llegar a conclusiones erradas, o al menos contradictorias, como las observadas en estudios previos que relacionan el RA con la $\mathrm{AE}$, ya que es probable que las correlaciones débiles o nulas se deban en mayor grado al método utilizado para operacionalizar el RA (Chapell et al., 2005; DordiNejad et al., 2011; Khalaila, 2015; Akram y Mahmood, 2010; Shakir, 2014).

Se concluye, entonces - al menos provisionalmente-, que no sería conveniente considerar indistintamente el RE y el PP cuando se relacionan con constructos específicos a una situación en particular, como la AE. En estos casos, parece más coherente utilizar el RE.

Con todo, cabe precisar que el presente estudio es de carácter exploratorio, por lo que los hallazgos deben ser interpretados con cautela. Del mismo modo, algunos aspectos pudieron influir en los resultados, tales como la evaluación de un grupo con una mayor proporción de mujeres (situación muy frecuente en las carreras de psicología en Perú), o bien un tamaño muestral relativamente pequeño y de una sola carrera profesional, por lo que en posteriores estudios deben subsanarse, a fin de llegar a conclusiones más sólidas. 


\section{Referencias}

Aiken, L. (1996). Tests psicológicos y evaluación (8a Ed.). Ciudad de México: Prentice Hall.

Akram, R. y Mahmood, N. (2010). The relationship between test anxiety and academic achievement. Bulletin of Education and Research, 32(2), 63-74.

Araki, N., Iwawaki, S. y Spielberger, C. D. (1992). Construction and validation of a Japanese adaptation of: the Test Anxiety Inventory. Anxiety, Stress and Coping: An International Journal, 5, 217-224. doi: 10.1080/10615809208249523

Ato, M., López, J. y Benavente, A. (2013). Un sistema de clasificación de los diseños de investigación en psicología. Anales de Psicología, 29(3), 1038-1059. doi: 10.6018/analesps.29.3.178511

Barrero, J., Garzón, G. y Gómez, Ó. (2013). Variables asociadas con el fenómeno de la deserción de los estudiantes en la Fundación Universitaria Konrad Lorenz. Pensando Psicología, 9(16), 55-68. doi: 10.16925/pe.v9i16.617

Cassady, J. C. y Johnson, R. E. (2001). Cogntive test anxiety and academic performance. Contemporary Educational Psychology, 27, 270-295. doi: 10.1006/ceps.2001.1094

Chapell, M. S., Blanding, Z. B., Silverstein, M. E., Takahashi, M., Newman, B., Gubi, A. y McCann, N. (2005). Test anxiety and academic performance in undergraduate and graduate students. Journal of Educational Psychology, 97(2), 268-274. doi: 10.1037/0022-0663.97.2.268

Cohen, J. (1992). A power primer. Psychological Bulletin, 112(1), 155-159.

Cortada de Kohan, N. (1999). Teorías psicométricas y construcción de tests. Buenos Aires: Lugar Editorial.

Domínguez-Lara, S. (2016a). Inventario de la Ansiedad ante Exámenes-Estado: análisis preliminar de validez y confiabilidad en universitarios de Lima. Liberabit, 22(2), 219-228.

Domínguez-Lara, S. (2016b). Sobre el uso de la prueba de significación de la hipótesis nula en la investigación psicológica. Cultura, 30, 141-150.

Domínguez-Lara, S., Calderón-de la Cruz, G., Alarcón-Parco, D. y Navarro-Loli, J. (en prensa). Relación entre ansiedad ante exámenes y rendimiento en exámenes en universitarios: análisis preliminar de la diferencia según asignatura. Revista Digital de Investigación en Docencia Universitaria.

DordiNejad, F., Hakimi, H., Ashouri, M., Dehghani, M., Zeinali, Z., Daghighi, M. y Bahrami, N. (2011). On the relationship between test anxiety and academic per- formance. Procedia, Social and Behavioral Sciences, 15, 3774-3778. doi: 10.1016/j.sbspro.2011.04.372

Edel, R. (2003). El rendimiento académico: concepto, investigación y desarrollo. Revista Electrónica Iberoamericana sobre Calidad, Eficacia y Cambio en Educacion, 1(2), 1-15.

Ferguson, C. J. (2009). An effect size primer: a guide for clinicians and researchers. Professional Psychology: Research and Practice, 40(5), 532-538. doi: 10.1037/ a0015808

Jiménez, M. (1994). Competencia social: intervención preventiva en la escuela. Infancia y Sociedad, 24, 21-48.

Khalaila, R. (2015). Relationship between academic self-concept, instrinsic motivation, test anxiety, and academic achievement among nursing students: Mediating and moderating effects. Nurse Education Today, 35, 432438. doi: 10.1016/j.nedt.2014.11.001

Paul, S. R. (1989). Test for the equality of several correlation coefficients. The Canadian Journal of Statistics, 17(2), 217-227. doi: 10.2307/3314850

Pizarro, R., Clark, L. y Allen, M. (1987). El ambiente educativo en el hogar. Diálogos Educacionales, 9, 66-83.

Sánchez-Rosas, J., Takaya, P. B. y Molinari, A. V.(2016). The Role of Teacher Behavior, Motivation and Emotion in Predicting Academic Social Participation in Class. Pensando Psicología, 12(19), 39-53. doi: 10.16925/pe.v12i19.1327

Seipp, B. (1991). Anxiety and academic performance: a meta-analysis of finding. Anxiety Research, 4(1), 27-41. doi: 10.1080/08917779108248762

Shakir, M. (2014). Academic anxiety as a correlate of academic achievement. Journal of Education and Practice, 5(10), 1-9.

Silver, N. C., Ullman, J. R. y Picker, C. (2015). COMPCOR: A computer program for comparing correlations using confidence intervals. Psychology and Cognitive Sciences, 1, 26-28. doi: 10.17140/PCSOJ-1-104

Silver, N. C. y Merino-Soto, C. (2016). Programas informáticos para comparaciones entre correlaciones: Muestras independientes. Revista Digital de Investigación en Docencia Universitaria, 10(1), 28-33. doi: 10.19083/ ridu. 2016.461

Silver, N. C., Zaikina, H., Hittner, J. B. y May, K. (2008). InCor: A computer program for testing differences among independent correlations. Molecular Ecology Resources, 8(4), 763-764. doi: 10.1111/j.1755-0998.2008.02107.x

Spielberger, C. D. (1980). Tensión y Ansiedad. Ciudad de México: Harla. 
Spielberger, C. D. y Vagg, P. (1995). Test Anxiety. A transactional process. En C. Spielberger y P. Vagg (Eds.), Test Anxiety: Theory, assessment and treatment (pp. 3-14). Washington: Taylor \& Francis.

Tooranposhti, M. G. (2011). A new approach for test anxiety treatment, academic achievement and met cognition. International Journal of Information and Education Technology, 1(3), 221-230. doi: 10.7763/IJIET.2011. V1.36
Vázquez, S. M., Noriega, M. y García, S. M. (2013). Relaciones entre rendimiento académico, competencia espacial, estilos de aprendizaje y deserción. Revista electrónica de investigación educativa, 15(1), 29-44.

Vázquez, S. y Daura, F. (2013). Auto-regulación del aprendizaje y rendimiento académico. Estudios pedagógicos (Valdivia), 39(1), 305-324. doi: 10.4067/ S0718-07052013000100018 